REVIEW

\title{
Incidental radiological diagnosis of rickets
}

\author{
L M Rennie, T F Beattie, A G Wilkinson, P Crofton, L E Bath
}

Emerg Med J 2005;22:534-537. doi: 10.1136/emj.2003.011668

Rickets fortunately remains rare in the United Kingdom, although its actual incidence is currently undetermined.' Many still consider it to be a disease of poverty prevalent during the Victorian era. However, a number of recent articles have highlighted concern among British health professionals about the number of cases still being diagnosed in this country. ${ }^{2-6}$ These cases have nearly all involved non-Caucasian children who are considered to be at high risk due to skin colour, prolonged breast feeding, and low maternal vitamin $D$ levels. Their presentations are variable ranging from failure to thrive, bone deformities, seizures, and even stridor. The diagnosis is usually made in babies and toddlers. We present a series of patients attending our accident and emergency (A\&E) department, over a five month period, where the diagnosis of rickets was primarily a radiological diagnosis.

See end of article for authors' affiliations

Correspondence to:

Correspondence to:
L Rennie, Accident and Emergency Department Royal Hospital for Sick Children, Sciennes Road, Edinburgh EH9 1LF,

Scotland; lovise.rennie@ kierto.com

Accepted for publication 4 August 2004 (fig 2). This did not demonstrate respiratory pathology but the appearance of the bones was thought to be unusual and the diagnosis of confirmed this the next working day, and the patient returned for blood tests in A\&E. These were in keeping with the diagnosis of rickets (see table 1). He was referred for treatment in the endocrine clinic.

Further examination revealed rachitic rosary, thickened wrists, and a large anterior fontanel. His weight was well below the 0.4th centile and head circumference between the 50th and the 90th centiles. He was treated with ergocalciferol 1500 units daily for four weeks and then 750 units daily for four weeks. Repeat blood tests after treatment had normalised.

This boy had been born in the UK to parents from the Middle East. He was still breast fed with a small amount of solids and little exposure to sunshine.

\section{Case 3}

A six month old boy was referred by his GP with pain in his right leg. Apparently his older brother had pulled his leg to free it from a baby seat and he had cried on manipulation of that leg since. An $x$ ray of his femur demonstrated a distal femoral fracture in addition to subtle changes of rickets (fig 3). Although the history was unusual and in other circumstances may have prompted investigation for non-accidental injury, the attending doctors felt that the fracture was pathological and consistent with the story of minimal trauma. A full leg cast was applied and his parents were given follow up appointments for both the orthopaedic and endocrine clinics. The patient was requested to re-attend A\&E to have blood taken after consultation with the endocrine team so that the results would be ready by the time of the clinic appointment (see table 1). In the interim, his mother had already commenced her son on vitamin drops containing vitamin D. This accounts for his $25(\mathrm{OH})$ vitamin D level being within the normal range, although his other blood parameters strongly suggest rickets. Further examination showed his weight to be on the 10th centile and his height on the 3rd centile. He also displayed rachitic rosary and splaying of his metaphyses. He was treated with ergocalciferol 1500 units daily for four weeks followed by 750 units daily for four weeks and his blood results subsequently returned to normal. He had been born in the UK to parents from Saudi Arabia and was still being exclusively breast fed.

\section{Case 4}

An 18 month old boy was brought to A\&E because of concern about his abnormal leg rickets was suspected. A consultant radiologist 


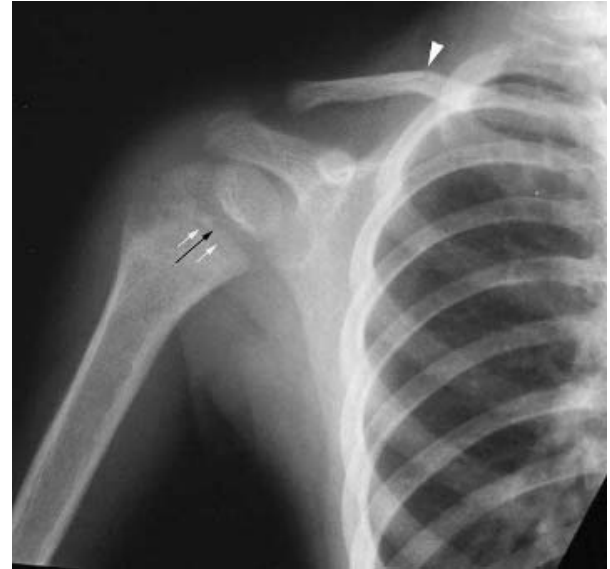

Figure 1 Radiograph of the shoulder and upper arm showing greenstick fracture of the clavicle (white arrowhead). Metaphyseal lucent line in the humerus (white arrows) indicates previous severe rickets. However, this has now improved with some bone formation adjacent to the growth plate (black arrow). Decreased bone density, coarsened trabecular pattern, and cortical tunnelling indicate secondary

hyperparathyroidism. Written permission was obtained from the parents of the child to have their clinical details published.

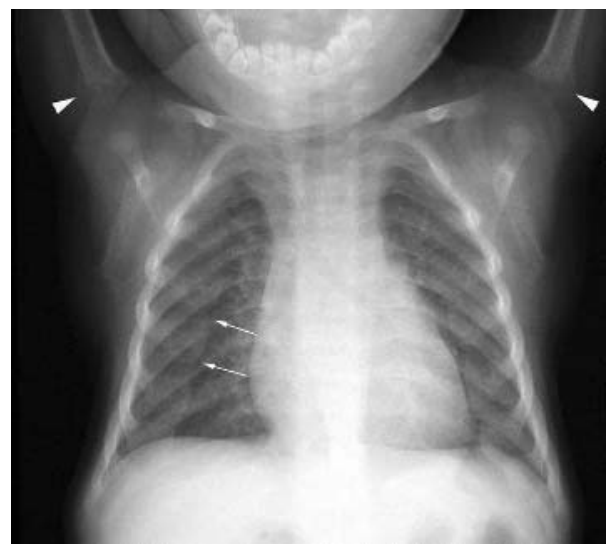

Figure 2 Chest radiograph showing expansion of the anterior rib ends (arrows), marked reduction in bone density, cortical tunnelling and fraying, and splaying of the proximal humeral growth plate (arrowheads). Written permission was obtained from the parents of the child to have their clinical details published.

shape. Examination revealed wrist swelling and rachitic rosary in addition to marked bowing of his legs. His weight was found to be on the 3rd centile and his height below the 3rd centile. Bilateral tibia and fibula $x$ rays (fig 4) were performed and a radiological opinion confirmed that the appearance was of rickets. An $x$ ray taken six months previously to exclude a finger fracture was then re-examined

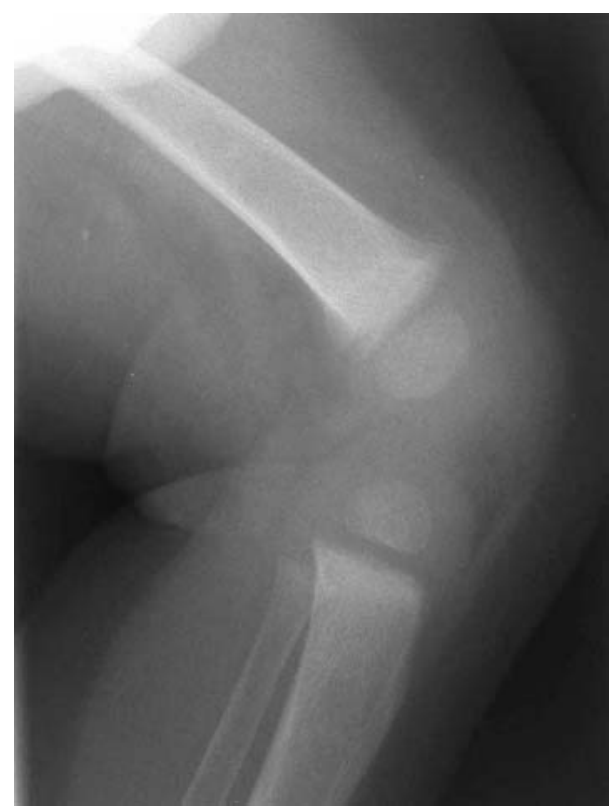

Figure 3 Lateral view of the knee demonstrating a green stick fracture of the distal femur. In addition, there is decreased bone density with loss of cortical definition and slight splaying of the metaphysis. The growth plate margin lacks clarity. Written permission was obtained from the parents of the child to have their clinical details published.

and in retrospect also showed changes of rickets (fig 5). Baseline blood investigations were performed that were consistent with rickets (see table 1). Follow up was arranged in the endocrine clinic. He was treated with ergocalciferol 3000 units daily for four weeks and then 1500 units daily for four weeks and 750 units for a final four weeks. His blood results were within normal limits after this time.

He was born in the UK to parents from Sudan. He had been breast fed with weaning introduced at four months but was rarely outside.

\section{DISCUSSION}

Rickets is due to the failure of osteoid calcification in a growing child and usually occurs as a result of vitamin D metabolite deficiency. Vitamin D (cholecalciferol) is formed in the skin after exposure to ultraviolet light. Human milk contains very little vitamin D and it follows that breastfed children are at increased risk of vitamin $\mathrm{D}$ deficiency. In addition, those with darkly pigmented skin or who cover their bodies are also at increased risk. Vitamin D is converted to $25(\mathrm{OH})$ vitamin $\mathrm{D}$ (calcidiol) in the liver. Plasma concentration of $25(\mathrm{OH})$ vitamin $\mathrm{D}$ is a reflection of vitamin D status derived from both endogenous and exogenous sources. In the kidney, $25(\mathrm{OH})$ vitamin D is converted to the active hormone $1,25(\mathrm{OH})_{2}$ vitamin $\mathrm{D}$ (calcitriol), which increases the concentrations of calcium and phosphate in

Table 1 Biochemistry of the four cases

\begin{tabular}{|c|c|c|c|c|c|}
\hline & $25(\mathrm{OH})$ vitamin $D(\mathrm{nmol} / \mathrm{l})$ & $\begin{array}{l}\text { Alkaline phosphatase } \\
\text { (units/l) }\end{array}$ & Calcium (mmol/l) & Phosphate (mmol/l) & $\begin{array}{l}\text { Parathyroid hormone } \\
\text { (ng/l) }\end{array}$ \\
\hline & Ref R: $15-100$ & Ref R: 180-900 & Ref R: 2.0-2.6 & Ref R: 1.0-2.1 & Ref R: 11-35 \\
\hline Case 1 & Sample not available & 2591 & 2.05 & 0.74 & 548 \\
\hline Case 2 & $<15$ & 6652 & 1.62 & 0.67 & 163 \\
\hline Case 3 & 26 & 2090 & 2.26 & 1.27 & 393 \\
\hline Case 4 & 12 & 1394 & 2.44 & 1.32 & Not done \\
\hline
\end{tabular}




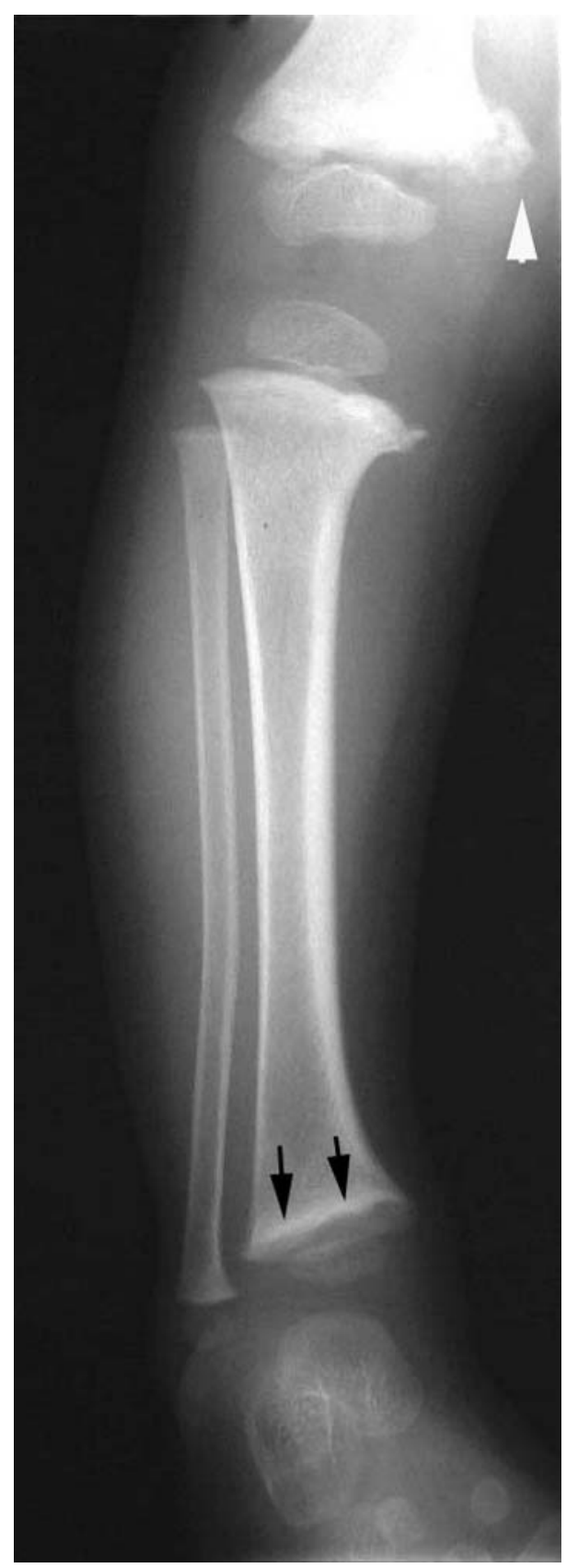

Figure 4 Radiograph of the lower limbs showing genu varum, fraying and splaying of the metaphyses with fragmentation of the medial aspect of the lower femoral metaphysis (white arrowhead). Dense zone of provisional ossification (black arrows) indicates that this is intermittent rickets currently in a healing phase. Written permission was obtained from the parents of the child to have their clinical details published.

extracellular fluid leading to the calcification of osteoid. When calcitriol levels are low, hypocalcaemia develops. This stimulates parathyroid hormone (PTH) and some of the changes seen in bone are those of hyperparathyroidism. Early in the course of the rickets, the calcium concentration in serum decreases. However, after stimulation of PTH, calcium returns to the normal range but phosphate usually falls. Alkaline phosphatase, produced by very active differentiated osteoblasts, is released into the extracellular fluid resulting in elevated serum levels.

In the paediatric A\&E setting, $x$ rays are largely performed to diagnose fractures or respiratory illness. Occasionally, other important pathology can be diagnosed incidental to the

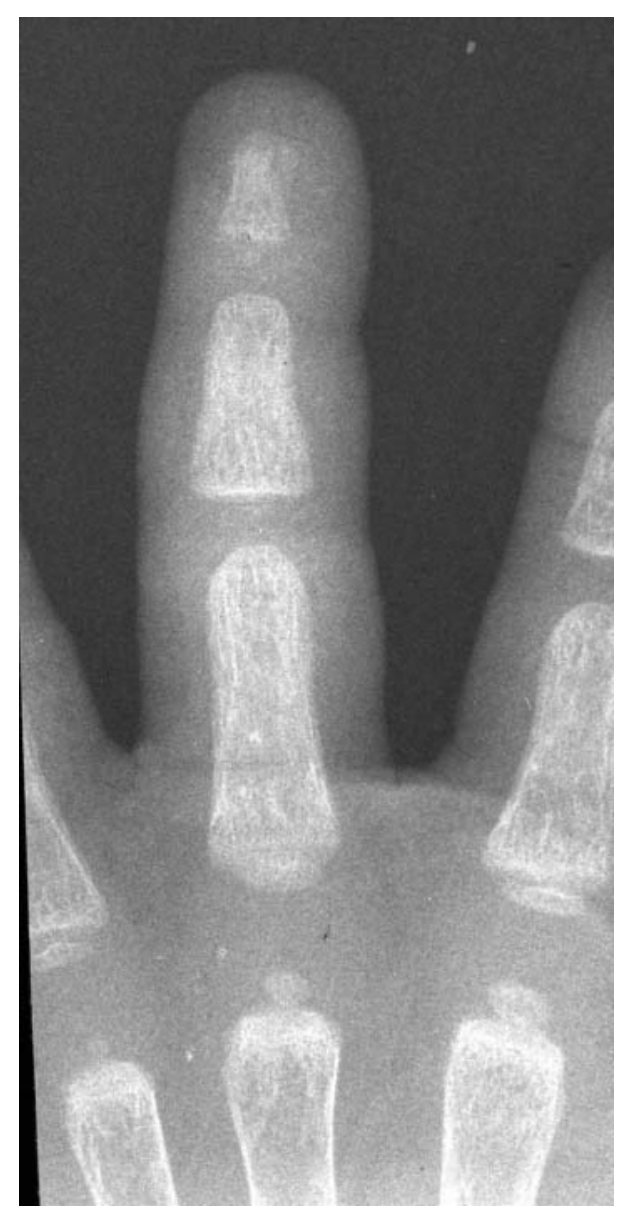

Figure 5 Radiograph of finger showing decreased bone density, coarsening of trabecular pattern, and cortical erosion indicating hyperparathyroidism. Written permission was obtained from the parents of the child to have their clinical details published.

presenting complaint. In three of our four cases the diagnosis of rickets was not suspected before an $x$ ray was performed (and in the fourth case the diagnosis could have been made six months earlier when the finger radiograph was taken). Although the $x$ rays were noted to have an abnormal appearance, the final diagnosis was made by a consultant radiologist. We are fortunate to have all our $x$ rays reported by a senior radiologist on the same or next working day. These cases illustrate the importance of rapid reporting of $x$ rays by senior radiology personnel in an A\&E department.

Our cases support previous findings that vitamin D deficient rickets occurs mainly in young, non-Caucasian children who are breast fed. ${ }^{2}{ }^{5}$ Vitamin D supplementation was not given to any of these children prior to presentation. This is contrary to the recommendation by the Department of Health's Committee on Medical Aspects of Food Policy (COMA), which states that supplementation should be given to all children up to the age of 3 years and up to 5 years if considered high risk of developing vitamin D deficiency. ${ }^{7}$ Public health was alerted to these cases through the children's health visitors.

Mughal et $a l^{2}$ questioned the historical assumption that rickets is associated with poverty. In keeping with this, our cases were mainly from well educated and relatively affluent families.

It has been postulated that Asian populations may have a genetic tendency to low vitamin $\mathrm{D}$ levels as a result of increased 24-hydroxylase activity. This theory may explain high levels of vitamin D deficiency in Asian populations even 
in areas where sunshine is plentiful. ${ }^{4}$ However, inadequate exposure to ultraviolet irradiation is still considered an important factor in the causation of vitamin D deficiency. This has prompted the suggestion that vitamin D deficiency is increased in Muslim women (and therefore their offspring) who wear concealing traditional dress, have dark skin, and spend a lot of time indoors. ${ }^{8} 9$ Three of the children were born to Muslim parents.

All our cases presented between the months of August to January, with an additional two cases noted in the hospital during this period. The preceding summer had been perceived to be particularly wet. This is perhaps borne out by the A\&E department's unusually low injury rate during this summer because it has been shown there appears to be a link between warm sunny weather and higher attendance with paediatric injuries. ${ }^{10}$ We have considered the possibility that bad weather during the summer months further depleted vitamin $\mathrm{D}$ levels in a vulnerable population and led to the unprecedented number of rickets cases presenting to our department. However, we cannot exclude other factors, such as an increase in the ethnic population or, indeed, mere chance.

Our A\&E department serves a population of 120000 children (under the age of 13). A total of 29000 new attendances are seen each year. Unfortunately we do not record how many of these attendances are children from ethnic backgrounds at increased risk of rickets, but it is a definite minority. It was therefore particularly striking for our department to have seen four cases of rickets in a five month period.

There have been a number of recent publications commenting on what is suggested to be a possible resurgence of vitamin $\mathrm{D}$ deficiency in non-Caucasian babies and toddlers. ${ }^{111}$ Despite the growing concern amongst medical professionals there have been no widespread public health interventions to protect non-Caucasian children from a preventable disease, such as the successful Stop Rickets Campaign in the 1980s. ${ }^{4}$ Paul Boateng, Undersecretary of State for Health in 1997, cautioned "against relying on anecdotal reports of rickets in the medical literature" because "there is no reliable evidence that rickets is on the increase, and we have evidence to show it is declining". This has certainly not been our experience in Edinburgh and we have found no medical literature to support a recent decline in rickets, although admittedly the literature does mainly contain case reports. Perhaps if rickets were a notifiable disease there would be figures on a national level. Our case series is, to the best of our knowledge, the first report from Scotland since the mid-eighties. ${ }^{12}$

\section{CONCLUSION}

The greatest improvements in reduction of this preventable disorder lie within the remit of public health professionals and politicians. However, clinicians should be alerted to become extra vigilant when seeing non-Caucasian children in A\&E. Our cases of incidental, radiologically detected rickets suggest there are probably a number of children in the community who remain undiagnosed.

\section{Authors' affiliations \\ L M Rennie, T F Beattie, Department of Accident and Emergency \\ Medicine \\ A G Wilkinson, Department of Radiology \\ P Crofton, Department of Paediatric Biochemistry \\ L E Bath, Department of Medical Paediatrics, Royal Hospital for Sick \\ Children, Edinburgh, Scotland \\ Funding: none. \\ Competing interests: none declared \\ Written permission was obtained from the parents of all children to have their clinical details published.}

\section{REFERENCES}

1 Ashraf S, Mughal MZ. The prevalence of rickets among non-Caucasian children. Arch Dis Child 2002;87:263--a.

2 Mughal MZ, Salama H, Greenaway T, et al. Lesson of the week: florid rickets associated with prolonged breast feeding without vitamin $D$ supplementation. BMJ 1999;318:39-40.

3 Zlotkin S, Blumsohn A. Vitamin D concentrations in Asian children living in England. BMJ 1999;318:1417.

4 Shaw NJ, Pal BR. Vitamin D deficiency in UK Asian families: activating a new concern. Arch Dis Child 2002;86:147-9.

5 Coren M. Re: vitamin D deficiency in UK Asian families: activating a new concern. BMJ 2003;86:147-9.

6 Wharton BA. Low plasma vitamin D in Asian toddlers in Britain. BMJ 1999;318:2-3.

7 Department of Health. Nutrition and one health; with particular reference to calcium and vitamin D. no.49. 1998. HMSO. Report on health and social subjects.

8 Guzel R, Konzanoglu E, Guler-Uysal F, et al. Vitamin D status and bone mineral density of veiled and unveiled Turkish women. J Womans Health Gend Based Med 1 A.D.; 10:765-70

9 Bachrach S, Fisher J, Parks JS. An outbreak of vitamin D deficiency rickets in a susceptible population. Pediatrics 1979;64:871-7.

10 Macgregor DM. Effect of weather on attendance with injury at a paediatric emergency department. Emerg Med J 2003;20:204.

11 Lawson $M$, Thomas $M$. Vitamin D concentrations in Asian children aged 2 years living in England: population survey. BMJ 1999;318:28.

12 Dunnigan MG, Gleiken BM, Henderson JB, et al. Prevention of rickets in Asian children: assessment of the Glasgow campaign. BMJ 1985;291:239-42. 